\title{
Pediatric living donor liver transplantation with homograft replacement of retrohepatic inferior vena cava for advanced hepatoblastoma
}

\author{
Jung-Man Namgoong ${ }^{1, *}$, Jin-Uk Choi ${ }^{1, *}$, Shin Hwang ${ }^{1}$, Suk-Hee $\mathrm{Oh}^{2}$, and Gil-Chun Park ${ }^{1}$ \\ Departments of ${ }^{1}$ Surgery and ${ }^{2}$ Pediatrics, Asan Medical Center, University of Ulsan College of Medicine, Seoul, Korea
}

\begin{abstract}
Replacement of the retrohepatic inferior vena cava (IVC) after concurrent resection of IVC and hepatocellular carcinoma-containing liver is settled as a feasible living donor liver transplantation (LDLT) technique to cope with tumors around the IVC. This technique makes LDLT comparable to deceased-donor liver transplantation (DDLT). In the current Korean setting, the common substitute for IVC is a Dacron graft for adult recipients. In contrast, such a synthetic graft cannot be used for pediatric patients because of ongoing growth. We present one pediatric LDLT case with IVC homograft replacement for advanced hepatoblastoma. The patient was a 8 year-old boy suffering from large multiple hepatoblastomas. The tumors encroached the retrohepatic IVC. Thus there was high risk of residual tumor cells at the IVC, if it was preserved. Thus, we decided to replace IVC at the time of LDLT. After waiting for $>1$ month, we finally obtained cold-stored IVC homograft and LDLT was performed with the mother's left liver. A $4 \mathrm{~cm}$-long IVC allograft was anastomosed at the back table. The left liver graft with IVC interposition was implanted along standard procedure similar to DDLT. The patient recovered uneventfully and is undergoing scheduled adjuvant chemotherapy. We have performed $>20$ cases of IVC replacement in adult recipients with hepatocellular carcinoma or Budd-Chiari syndrome, but all vessel substitutes were synthetic, because sizable IVC homograft is unavailable. In pediatric recipients, various vein homografts such as iliac vein, IVC and other large-sized veins, can be used depending on body size of recipient and availability of vessel grafts. (Ann Hepatobiliary Pancreat Surg 2019;23:178-182)
\end{abstract}

Key Words: Interposition; Venoplasty; Pediatric transplantation

\section{INTRODUCTION}

Replacement of the retrohepatic inferior vena cava (IVC) after concurrent resection of IVC and hepatocellular carcinoma (HCC)-containing liver is settled as an optional technique of living donor liver transplantation (LDLT) to cope with HCCs around the IVC. ${ }^{1-4}$ This technique makes the resection extent of LDLT comparable to that of deceaseddonor liver transplantation (DDLT). In the current Korean setting, the common substitute for IVC is a Dacron graft for adult recipients. In contrast, such a synthetic graft cannot be used for pediatric patients because of ongoing physical growth. Thus, homograft should be used. There are only few cases on IVC replacement in pediatric LDLT. ${ }^{5}$ We herein present one case of IVC replacement per- formed during pediatric LDLT to treat advanced hepatoblastoma.

\section{CASE}

The patient was an 8 year-old $28 \mathrm{Kg}$-weighing boy suffering from large multiple hepatoblastomas, partially responsive to systemic chemotherapy (Fig. 1). The tumors encroached the retrohepatic IVC. Thus, there was high risk of residual tumor cells attached at the IVC, if it was preserved (Fig. 2). Thus we decided to replace IVC at the time of LDLT operation. Since we did not have any cryopreserved IVC allograft as well as we were reluctant to use cryopreserved vein allograft for pediatric patients because of high risk of late aneurysmal weakening or shrink-

Received: April 9, 2019; Revised: April 12, 2019; Accepted: April 13, 2019

Corresponding author: Shin Hwang

Department of Surgery, Asan Medical Center, University of Ulsan College of Medicine, 88 Olympic-ro 43-gil, Songpa-gu, Seoul 05505, Korea Tel: +82-2-3010-3930, Fax: +82-2-3010-6701, E-mail: shwang@amc.seoul.kr

*These two authors contributed equally as first co-authors.

Copyright (C) 2019 by The Korean Association of Hepato-Biliary-Pancreatic Surgery

This is an Open Access article distributed under the terms of the Creative Commons Attribution Non-Commercial License (http://creativecommons.org/ licenses/by-nc/4.0) which permits unrestricted non-commercial use, distribution, and reproduction in any medium, provided the original work is properly cited. Annals of Hepato-Biliary-Pancreatic Surgery • pISSN: 2508-5778 • elSSN: 2508-5859 

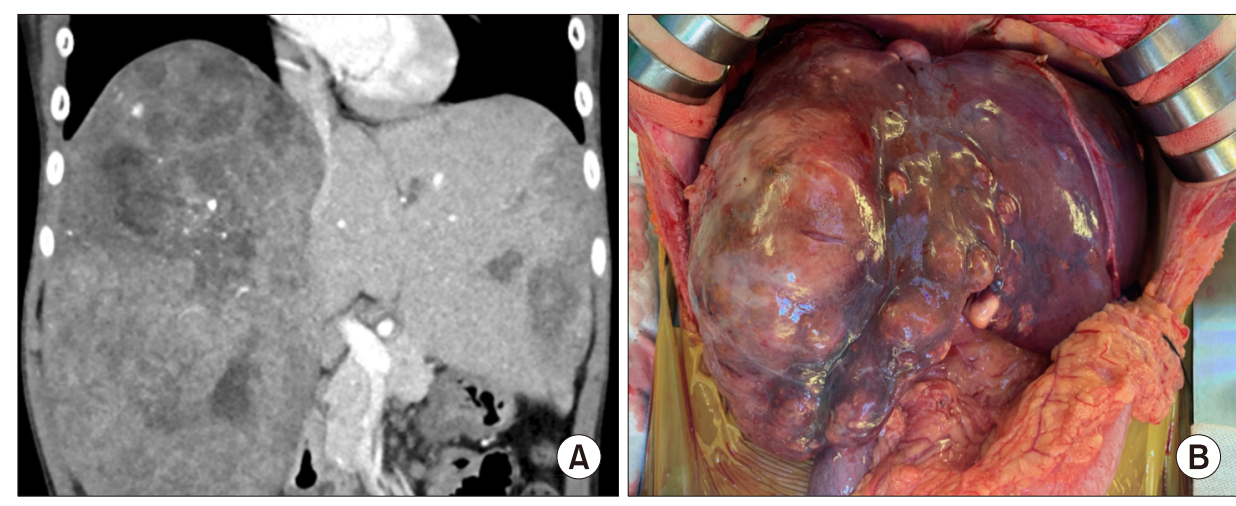

Fig. 1. Pretransplant computed tomography finding (A) and intraoperative photograph (B) of the recipient. Huge multiple tumors occupied the whole liver and the inferior vena cava is also invaded.
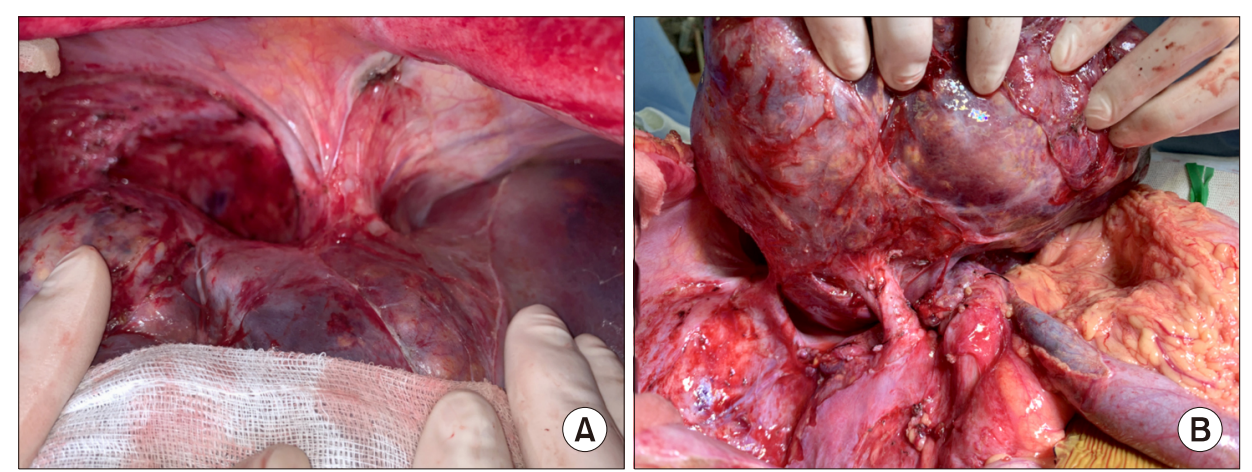

Fig. 2. Intraoperative photographs of the recipient shows tumor invasion of the cephalic (A) and caudal (B) parts of the inferior vena cava.
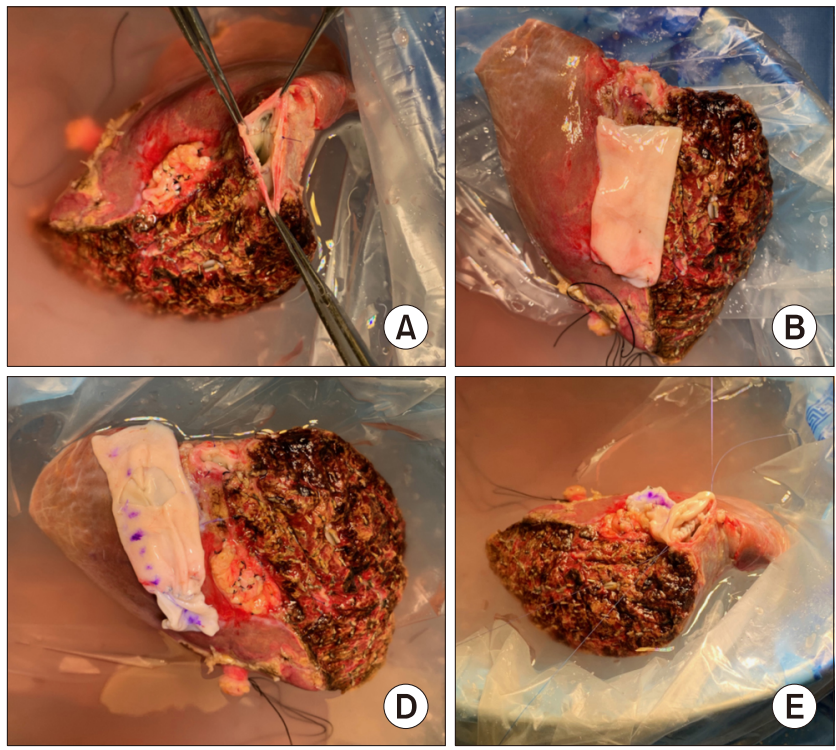

(E)

age, we waited until suitable large-sized vein allograft was available.

After waiting for more than one month, we finally obtained cold-stored IVC allograft, recovered from a deceased organ donor. A 250 g-weighing left liver graft was harvested from his 42 year-old mother and the $4 \mathrm{~cm}$-long IVC allograft was anastomosed at the back table. Since such a length of IVC allograft was too short to replace

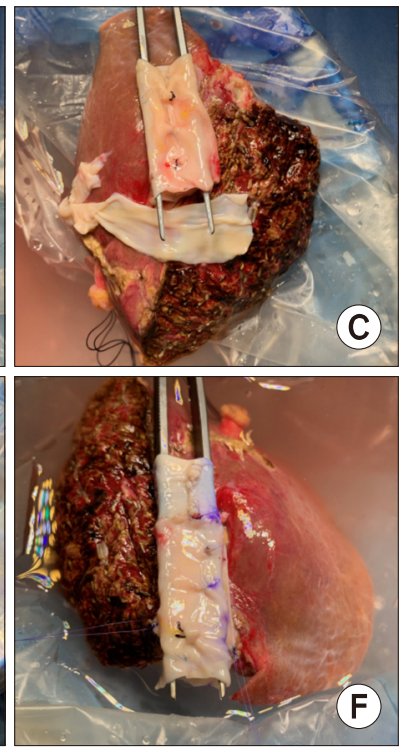

Fig. 3. Intraoperative photographs of the bench work. (A) The middle and left hepatic vein orifices are unified. (B) A $4 \mathrm{~cm}$ long cold-preserved vena cava homograft is placed to assess matching in size and length. $(\mathrm{C}$, D) Since the vena cava homograft appears short, another iliac vein homograft was attached to elongate its length. (E, F) Vena cava homograft is attached to the left liver graft. the resected recipient's IVC, another vein homograft patch was attached to elongate the IVC interposition graft (Figs. 3 and 4).

Recipient's native liver including the retrohepatic IVC was removed completely (Fig. 5). The left liver graft with IVC interposition was implanted along standard procedure (Fig. 6). After completion of reconstruction, the potentially tumor-invaded right diaphragm was excised, and repaired 


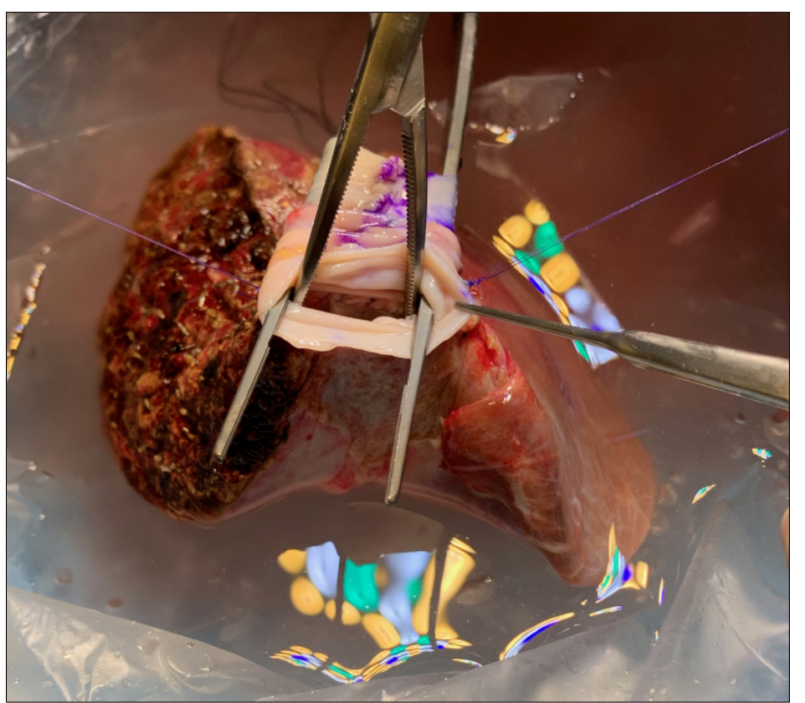

Fig. 4. Intraoperative photographs of the left liver graft combined with a vena cava homograft. The outflow orifice of the conjoined graft hepatic veins is visible. primarily (Fig. 7). Thereafter, indocyanine green (ICG) fluorescent imaging was applied to identify any remaining tumor, in which no abnormal fluorescent image was detected.

Since the liver graft was relatively very small compared with the 2064 g-weighing tumor-bearing native liver, a tissue expander was placed to prevent rotation toward the right subphrenic fossa. The tissue expander was removed after 2 weeks (Fig. 8).

This patient recovered uneventfully and is doing well for 3 months to date. He is currently undergoing scheduled adjuvant chemotherapy.

\section{DISCUSSION}

Compared with DDLT, LDLT has a definite demerit on
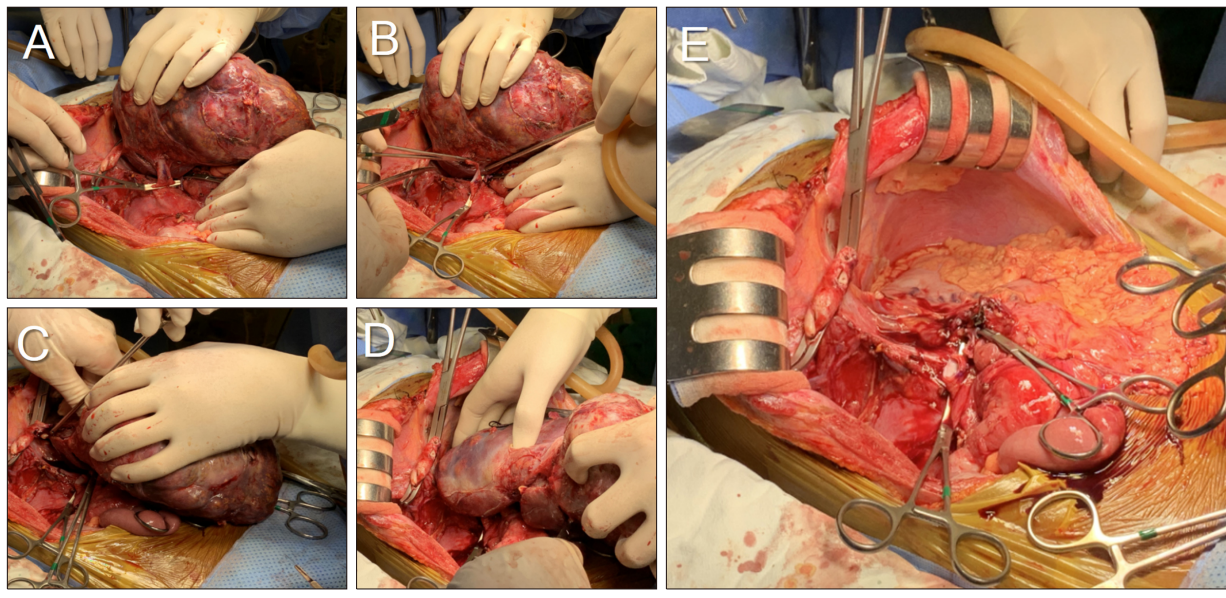

Fig. 5. Intraoperative photographs of the recipient hepatectomy. (A-D) The retrohepatic vena cava was excised after clamping. (E) The native liver is removed completely.
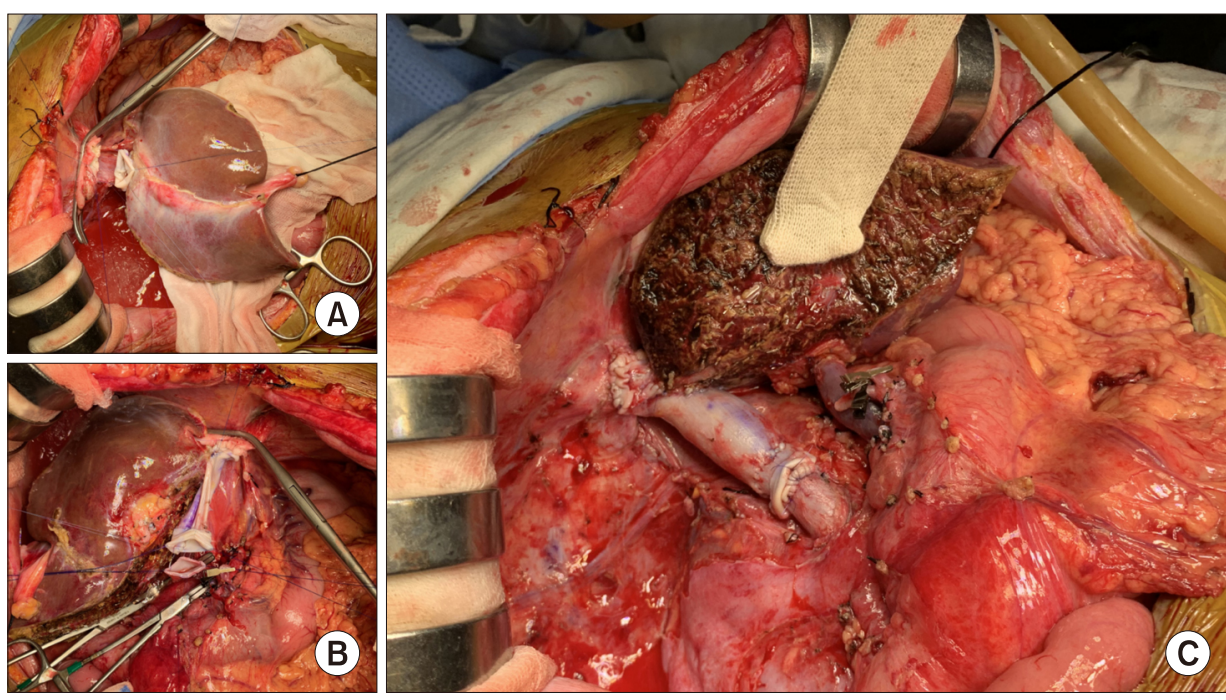

Fig. 6. Intraoperative photographs of the graft implantation. (A, B) The cephalic and caudal ends of the vena cava homograft were anastomosed using 5-0 PDS. (C) The liver graft is reperfused after portal vein reconstruction. 


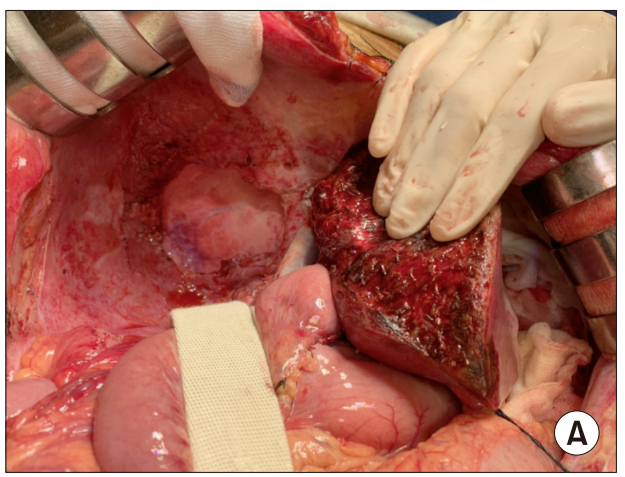

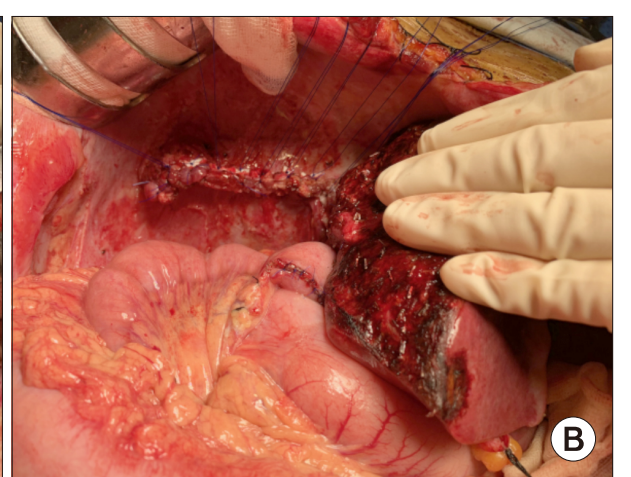

B)
Fig. 7. Intraoperative photographs of the diaphragm excision. The tumor-invaded diaphragm was excised (A) and repaired directly (B).
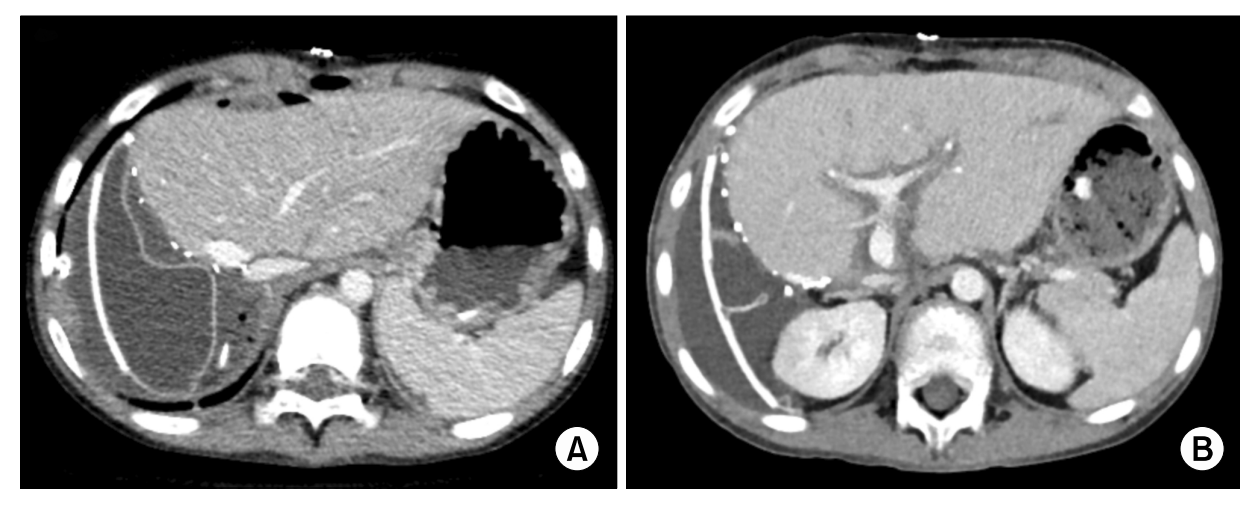

Fig. 8. Posttransplant computed tomography finding showing tissue expander support at day 4 and its decompression at day 12 after transplantation. oncological aspect in patients with hepatic malignancy because the recipient IVC should be preserved. If the IVC can be removed concurrently during LDLT operation, the extent of hepatectomy becomes comparable to that of DDLT. In clinical practice for LDLT, IVC replacement has been performed for the patients with HCCs closely located to the IVC or Budd-Chiari syndrome. ${ }^{1-6}$

In the current Korean setting, the common substitute for IVC is Dacron graft for adult recipients. ${ }^{6}$ This synthetic vessel institutes provides a large diameter, which exceeds the usual diameter of normal IVC, thus it is suitable for partial liver implantation. However, such a synthetic graft cannot be used for pediatric patients because of ongoing growth. There have been only few reports on IVC replacement in pediatric LDLT. ${ }^{5}$ The biggest hindrance against IVC replacement is availability of sizable vein homograft in countries where pediatric LDLT is frequently performed but the incidence of deceased donors is not high.

After deciding to perform LDLT with IVC replacement in this case, we had to wait for more than one month to obtained a fresh cold-stored IVC homograft, which was recovered from a deceased organ donor. The homograft was retrieved after obtaining a written consent on tissue donation and registered to the tissue bank at our institution. At this time, we urgently scheduled the LDLT operation just after confirmation of negative bacteria culture finding. To use the IVC homograft in a fresh state, it can be stored in $4{ }^{\circ} \mathrm{C}$ within 7 days instead of cryopreservation. Since the length of $4 \mathrm{~cm}$ was too short to replace the whole length of IVC resection, this homograft was elongated with other patches of vein homograft.

In this case, we applied intraoperative ICG fluorescent imaging to identify any remaining tumor. Yamamichi et al reported their experience that ICG fluorescent imaging was useful for surgical navigation in 3 hepatoblastoma patients and concluded that intraoperative ICG fluorescence imaging for patients with hepatoblastoma was feasible and useful for identifying small viable lesions and confirming that no remnant tumor remained after resection. ${ }^{7}$ We suggest injecting ICG dye intravenously more than 24 hours before operation, to make ICG accumulate at the tumor tissue.

In this case, the abdominal cavity was distended due to enlarged liver with large multiple tumors, but the liver graft was much smaller than the liver size. To place the 
left liver graft in the right position, we inserted a tissue expander to support the liver graft. We are not reluctant to insert a tissue expander if indicated. Mechanical support using a tissue expander is one of the standard procedures for pediatric LDLT developed many years ago. ${ }^{8}$ If the partial liver graft is large enough compared with the abdominal cavity, a slight dextro-positioning of the graft can be beneficial to hemodynamics-compliant hepatic vein reconstruction as well as stable positioning of the liver graft.

In pediatric recipients, various vein homografts such as iliac vein, IVC and other large-sized veins can be used depending on body size of the recipient and availability of vessel graft. We emphasize cryopreserved veins should be avoided if possible for pediatric patients because they can degenerate over time. Sasaki et al presented IVC replacement using a cryopreserved IVC homograft in a 10 year-old pediatric patient with Budd-Chiari syndrome. ${ }^{5}$

In conclusion, IVC replacement with homograft is a feasible option of LDLT in pediatric patients with advanced liver malignancies.

\section{REFERENCES}

1. Chen CL, Concejero AM, Wang CC, Wang SH, Liu YW, Yang $\mathrm{CH}$, et al. Inferior vena cava replacement in living donor liver transplantation for hepatocellular carcinoma. Liver Transpl 2009; 15:1637-1640.

2. Matsuda H, Sadamori H, Shinoura S, Umeda Y, Yoshida R, Satoh D, et al. Aggressive combined resection of hepatic inferior vena cava, with replacement by a ringed expanded polytetrafluoroethylene graft, in living-donor liver transplantation for hepatocellular carcinoma beyond the Milan criteria. J Hepatobiliary Pancreat Sci 2010;17:719-724.

3. Moon DB, Lee SG, Hwang S, Kim KH, Ahn CS, Ha TY, et al. No-touch en bloc right lobe living-donor liver transplantation with inferior vena cava replacement for hepatocellular carcinoma close to retrohepatic inferior vena cava: case report. Transplant Proc 2013;45:3135-3139.

4. Zahr Eldeen F, Abdulfatah MR, Broering D. Inferior vena cava replacement in living-donor liver transplant for hepatocellular carcinoma: first case report in Saudi Arabia. Exp Clin Transplant 2018;16:625-627.

5. Sasaki K, Kasahara M, Fukuda A, Shigeta T, Tanaka H, Nakagawa S, et al. Living donor liver transplantation with vena cava reconstruction using a cryopreserved allograft for a pediatric patient with Budd-Chiari syndrome. Transplantation 2009; 87:304-305.

6. Yoon YI, Lee SG, Moon DB, Ahn CS, Hwang S, Kim KH, et al. Surgical techniques and long-term outcomes of living-donor liver transplantation with inferior vena cava replacement using atriocaval synthetic interposition graft for Budd-Chiari syndrome. Ann Surg 2019;269:e43-e45.

7. Yamamichi $T$, Oue $T$, Yonekura $T$, Owari M, Nakahata $K$, Umeda S, et al. Clinical application of indocyanine green (ICG) fluorescent imaging of hepatoblastoma. J Pediatr Surg 2015;50: 833-836.

8. Inomata $\mathrm{Y}$, Tanaka $\mathrm{K}$, Egawa $\mathrm{H}$, Uemoto $\mathrm{S}$, Kiuchi T, Satomura $\mathrm{K}$, et al. Application of a tissue expander for stabilizing graft position in living-related liver transplantation. Clin Transplant 1997;11:56-59. 